\title{
Modelo experimental de fundoplicatura gastroesofágica em ratos. Estudo manométrico e histológico do esôfago ${ }^{1}$
}

\author{
Sérgio Luiz Rocha ${ }^{2}$, Zacarias Alves de Souza Filho ${ }^{3}$, Francielle Fátima Borges ${ }^{4}$, Giselle Stroparo, \\ Michele Mamprim Grippa ${ }^{4}$, Patrícia Carla Zanellato Gonçalves ${ }^{4}$
}

\begin{abstract}
Rocha SL, Souza Filho ZA, Borges FF, Stroparo G, Grippa MM, Gonçalves PCZ. Modelo experimental de fundoplicatura gastroesofágica em ratos: estudo manométrico e histológico do esôfago. Acta Cir Bras [serial online] 2004 MarAbr;19(2). Disponível em URL: http://www.scielo.br/acb.

RESUMO - Objetivo: O presente estudo objetiva avaliar alguns aspectos decorrentes da fundoplicatura em ratos, com técnica de Nissen, através da análise histológica do esôfago distal e estudo manométrico do esfíncter esofágico inferior (EEI), bem como da avaliação da variação do peso dos ratos submetidos ao procedimento, com diferentes dias de evolução. Métodos: Utilizaram-se 70 ratos Wistar, machos, com idade entre 100 e 130 dias e peso entre 250 e $300 \mathrm{~g}$, divididos em dois grupos. O grupo experimento foi composto por 60 ratos, subdivididos em 6 subgrupos de acordo com o tempo de aferição manométrica pós-operatória: 02, 07, 14, 21, 28 e 40 dias, e submetido a fundoplicatura com posterior análise manométrica do EEI e histológica do esôfago distal. O grupo controle foi composto pelos 10 animais restantes, submetido apenas a laparotomia com manipulação da região esôfago-gástrica, e realizaram-se as mesmas análises. Resultados: Evidenciou-se na análise histológica, edema estatisticamente significativo $(\mathrm{p}=0,0000)$ nos subgrupos 02 e 07 dias, acompanhado de perda de peso. Observou-se significância estatística no estudo manométrico em todos os subgrupos comparados ao controle $(\mathrm{p}<0,0018)$. A correlação edema e peso foi linear significativa e inversamente proporcional $\left(\mathrm{t}=-2,577<\mathrm{t}_{\mathrm{c}}=2,132\right)$. Não se verificou correlação linear significativa entre manometria e peso. Estabeleceu-se correlação linear significativa e diretamente proporcional entre edema e manometria $(\mathrm{t}=+6,085>\mathrm{t}=+2,132)$. Conclusão: Após fundoplicatura de Nissen em ratos, a pressão no EEI não retorna aos níveis do controle até o $40^{\circ}$ pós-operatório. $\mathrm{O}$ edema correlacionado à perda de peso e ao aumento da pressão no EEI pode ser a principal variável relacionada à possível disfagia.
\end{abstract}

DESCRITORES - Fundoplicatura. Disfagia. Edema. Manometria.

\section{Introdução}

A doença do refluxo gastroesofágico (DRGE), a mais freqüente das afecções esofágicas, é uma condição comum e crônica.

A maioria dos pacientes com DRGE necessita de tratamento médico durante toda a vida, e alguns deles apresentam complicações graves, como úlcera, estenose esofágica e esôfago de Barret 1,2,3,4.

O tratamento cirúrgico é indicado para pacientes com complicações da DRGE ou com tratamento clínico ineficaz. Atualmente, a fundoplicatura gástrica é o procedimento cirúrgico disponível mais efetivo para o tratamento da DRGE ${ }^{5 .}$ Dentre as opções cirúrgicas existentes, a técnica de Nissen representa o procedimento "padrão-ouro" no tratamento desta afecção.

Entretanto, apesar de ser bastante utilizada, a cirurgia de Nissen não é livre de complicações pós-operatórias. Dentre estas, podem ser citadas disfagia, distensão gástrica, incapacidade para eructar e vomitar, dor epigástrica e flatulência ${ }^{6}$.

Após a fundoplicatura de Nissen, a disfagia é uma queixa freqüente, sendo mais comum durante os primeiros meses pós- operatórios, com tendência a desaparecer no decorrer do período. Alguns estudos relatam, como possíveis causas, alterações secundárias ao edema pós-operatório na região manipulada, embora seu mecanismo não esteja definido $7,8,9,10,11$.

O objetivo do presente estudo é avaliar as consequiências da fundoplicatura em ratos, com técnica de Nissen, através da análise histológica do esôfago distal e do estudo manométrico do esfíncter esofágico inferior, bem como da avaliação da variação do peso de ratos submetidos ao procedimento com diferentes dias de evolução.

1. Trabalho desenvolvido na Disciplina de Técnica Operatória e Cirurgia Experimental da Pontifícia Universidade Católica do Paraná (PUCPR).

2. Prof. Adjunto da Disciplina de Anatomia Médica da PUCPR e UFPR. Prof. Adjunto da Disciplina de Técnica Operatória e Cirurgia Experimental da PUCPR. Doutor em Clínica Cirúrgica pela UFPR.

3. Prof. Titular de Clínica Cirúrgica do CCBS-PUCPR e da Disciplina de Técnica Operatória e Cirurgia Experimental da PUCPR e Disciplina de Cirurgia Geral da UFPR.

4. Alunas de Iniciação Científica da Disciplina de Técnica Operatória e Cirurgia Experimental da PUCPR. 


\section{Métodos}

O presente estudo foi aprovado pelo Comitê de Ética de Pesquisa com Animais do Centro de Ciências Biológicas e da Saúde da Pontifícia Universidade Católica do Paraná-CEPA-CCBS - PUCPR.

Utilizaram-se 70 ratos Wistar (Rattus novergicus albinus, Rodentia mammalia) machos, com idade entre 100 e 130 dias e peso entre 250 e 300 gramas. Os animais permaneceram no Biotério Central da Pontifícia Universidade Católica do Paraná em caixas numeradas e padronizadas para a espécie, com número máximo de cinco ratos por caixa, sob temperatura e luminosidade naturais, recendo água potável e ração padrão comercial em regime livre.

Os animais foram divididos em 2 grupos, um grupo denominado "experimento", composto por 60 ratos, e o outro denominado "controle", composto por 10 ratos. Utilizou-se, para indução e manutenção anestésicas, éter etílico 97\% administrado por via inalatória. Os 60 animais do grupo "experimento" foram submetidos a laparotomia mediana e fundoplicatura gastroesofágica, com técnica de Nissen, utilizando 3 pontos de polipropileno 6.0 para confecção da válvula e, a seguir, foram divididos em 6 subgrupos, de acordo com o tempo de aferição manométrica pós-operatória: $02,07,14,21,28$ e 40 dias. Os 10 animais do grupo "controle" foram submetidos apenas a laparotomia e manipulação da região esôfago-gástrica e, o estudo manométrico foi realizado imediatamente após o procedimento cirúrgico.

Os ratos tiveram seus pesos aferidos até o dia pré-determinado de eutanásia conforme cada subgrupo.

A medida da pressão do esfíncter esofágico inferior foi realizada no momento posterior à eutanásia, objetivando excluir a interferência da variação de pressão intratorácica e intra-abdominal na aferição manométrica. A manometria foi feita com o transdutor de pressão de um monitor cardíaco digital multicanal (biomotor 7.0 marca Bese), adaptado a um catéter de três vias. A primeira via foi conectada a um frasco de solução salina isotônica, com gotejamento de $4 \mathrm{ml}$ por minuto. A segunda via foi utilizada para limpeza do sistema. A terceira via foi ligada ao transdutor de pressão, e este ao monitor.

Realizou-se cervicotomia anterior, com isolamento do esôfago e sob visibilização direta, foi possível a introdução, via oral, do catéter com o transdutor de pressão. O fluxo contínuo de soro através do catéter permitiu verificar o aumento gradativo dos valores pressóricos até o momento de estabilização de um nível de pressão. Este foi considerado como valor máximo, correspondendo à maior pressão suportada pelo EEI após a fundoplicatura.

A peça cirúrgica contendo a fundoplicatura foi removida para estudo anátomopatológico. O conjunto foi imerso em formaldeído $10 \%$ e os cortes histológicos foram corados com hematoxilina-eosina. O parâmetro microscópico analisado foi a presença de edema e sua quantificação. As lâminas foram analisadas através de microscopia óptica, em aumento de 40 vezes, com auxílio de lente objetiva graduada. O critério estabelecido para verificar a presença ou não de edema foi a distância da superfície do epitélio esofágico até o início da camada muscular. Foram efetuadas 30 aferições por lâmina, seguidas de cálculo da média dos valores obtidos. Esta

média foi multiplicada por 0,12 (valor prédeterminado para correção de dados quando utilizado aumento de 40 vezes) com a finalidade de conversão em micrômetros. Foram obtidos os mesmos registros nos animais do grupo controle.

O registro da variação do peso foi convertido em porcentagem para possibilitar o cálculo estatístico.

Os resultados dos estudos histológico e manométrico foram submetidos a tratamento estatístico com o teste " $\mathrm{t}$ " de Student e teste de Mann-Whitney. Para comparação entre variáveis diferentes foi utilizado o artifício da correlação linear. Considerou-se $p<0,05$ para rejeição da hipótese de nulidade.

\section{Resultados}

O parâmetro edema avaliado no estudo microscópico, bem como sua quantificação nos grupos controle e experimento (subgrupos 02, 07, 14, 21, 28 e 40 dias) estão descritos na Tabela 1.

TABELA 1 - Estudo microscópico. Quantificação do edema na região da fundoplicatura gastroesofágica (micrômetros).

\begin{tabular}{lcc}
\hline Grupos & Tempo de aferição & Quantificação do edema \\
\hline Grupo Controle & & $142,1 \mu$ \\
\hline Grupo Experimento & 2 dias & $1265,1 \mu$ \\
& 7 dias & $641,1 \mu$ \\
& 14 dias & $404,1 \mu$ \\
& 21 dias & $157,5 \mu$ \\
& 28 dias & $182,9 \mu$ \\
& 40 dias & $173,0 \mu$ \\
\hline
\end{tabular}

Grupo Controle x Grupo Experimento. Teste " $t$ " de Student

\begin{tabular}{|c|c|}
\hline 2 dias .................. & $\mathrm{p}=0,0000 *$ \\
\hline 7 dias & $\mathrm{p}=0,0000 *$ \\
\hline 14 dias . & $\mathrm{p}=0,1047$ \\
\hline 21 dias . & $\mathrm{p}=0,4731$ \\
\hline 28 dias ... & $\mathrm{p}=0,1941$ \\
\hline 40 dias & $\mathrm{p}=0,2762$ \\
\hline
\end{tabular}

* presença de diferença significativa

Foi evidenciada diferença estatisticamente significativa apenas nos subgrupos de 02 e 07 dias. Nos subgrupos a partir de 14 dias, os valores observados foram estatisticamente semelhantes. Quanto à comparação entre os grupos experimento (subgrupo 02 e 07) e controle, foi demonstrada evidente disparidade quanto ao estudo microscópico do edema, como mostrado nas Figuras 1 e 2. 


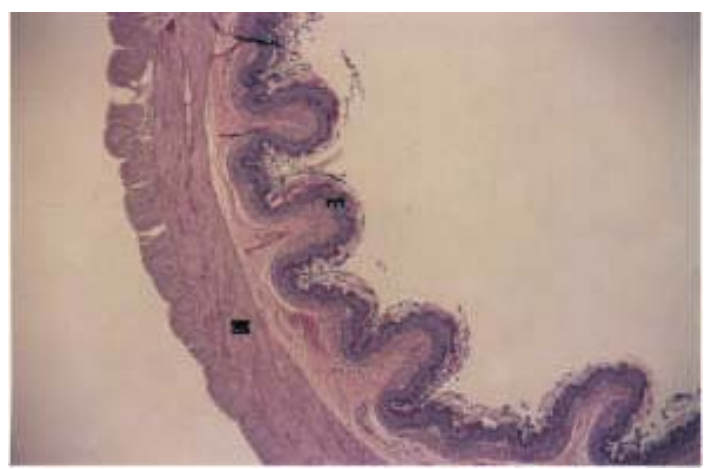

FIGURA 1 - Mucosa justaposta à muscular própria (Grupo controle - esôfago cortado transversalmente HEX40).

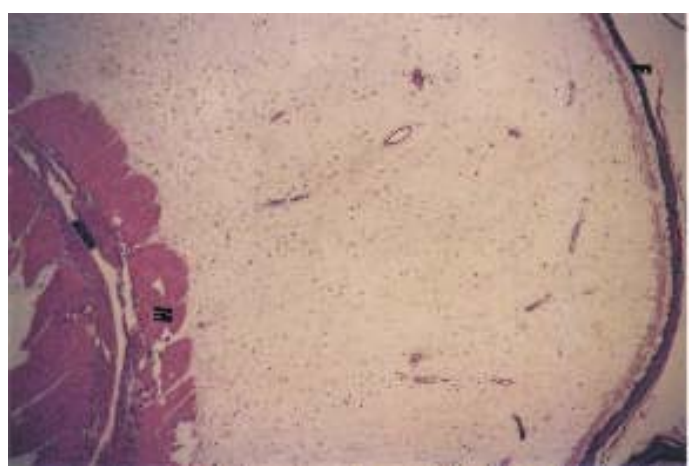

FIGURA 2 - Edema de submucosa (Subgrupo 02 dias esôfago cortado transversalmente HEX40).
A avaliação manométrica comparativa entre os grupos controle e experimento está descrita na Tabela 2.

Como os dados obtidos não foram totalmente adequados para o uso do teste " $\mathrm{t}$ " de Student, foi utilizado também o teste não- paramétrico de Mann-Whitney para obtenção de resultados mais consistentes. Nos dois testes realizados foi observada diferença estatisticamente significativa em todos os subgrupos do grupo experimento, quando comparados ao controle. Estudaram-se as associações entre as variáveis analisadas, ou seja, quantificação do edema, variação do peso e manometria do esfíncter esofágico inferior. Os dados estão descritos nas Tabelas 3, 4 e 5.

Como valor $\mathrm{t}=-2,577$ é menor que o valor $\mathrm{t}_{\mathrm{c}}=-2,132$, existe uma correlação linear

TABELA 2 - Estudo Manométrico. Pressão do esfíncter esofágico inferior (EEI).

\begin{tabular}{lcc}
\hline Grupos & Tempo de Aferição & Pressão no EEI \\
\hline Grupo Controle & $0,70 \mathrm{mmHg}$ & \\
Grupo Experimento & 2 dias & $10,07 \mathrm{mmHg}$ \\
& 7 dias & $5,07 \mathrm{mmHg}$ \\
& 14 dias & $3,30 \mathrm{mmHg}$ \\
21 dias & $4,00 \mathrm{mmHg}$ \\
28 dias & $3,50 \mathrm{mmHg}$ \\
& 40 dias & $2,57 \mathrm{mmHg}$ \\
\hline
\end{tabular}

Grupo Controle x Grupo Experimento. Teste "t" de Student Teste de Mann-Whitney

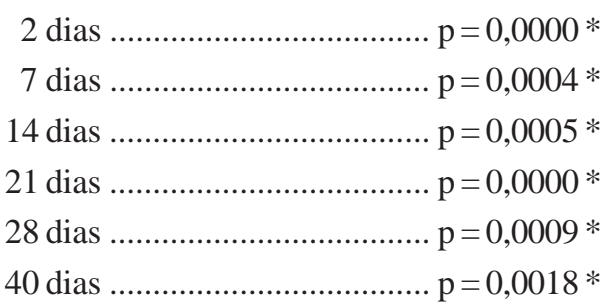

$\mathrm{p}=0,0000 *$

$\mathrm{p}=0,0003 *$

$\mathrm{p}=0,0016 *$

$\mathrm{p}=0,0000 *$

$\mathrm{p}=0,0016 *$

* presença de diferença significativa

significativa entre a variação de peso e edema.

O sinal negativo indica correlação inversa, ou seja, aumentando a variável edema iminui a variável peso e vice-versa.

Como valor $\mathrm{t}=-2,075$ é maior que o valor esfíncter esofágico inferior. com a variável manometria. 
TABELA 3 - Correlação entre variação do peso e edema.

\begin{tabular}{llrl}
\hline Grupos & $\begin{array}{l}\text { Tempo de } \\
\text { Aferição }\end{array}$ & Variação do peso & $\begin{array}{l}\text { Quantificação } \\
\text { do edema }\end{array}$ \\
\hline Grupo Experimento & 2 dias & $87,60 \%$ & $1265,1 \mu$ \\
& 7 dias & $83,70 \%$ & $641,1 \mu$ \\
& 14 dias & $101,40 \%$ & $404,1 \mu$ \\
& 21 dias & $102,80 \%$ & $157,5 \mu$ \\
28 dias & $105,30 \%$ & $182,9 \mu$ \\
& 40 dias & $113,50 \%$ & $173,0 \mu$ \\
\hline Coeficiente de correlação & & $\mathrm{r}=-0,790$ \\
Teste de significância (estatística do teste) & & $\mathrm{t}=-2,577$ \\
Valor crítico (nível de significância) & & $\mathrm{tc}=-2,132$
\end{tabular}

TABELA 4 - Correlação entre manometria e peso.

\begin{tabular}{llrr}
\hline Grupos & Tempo de Aferição & Manometria & Variação do peso \\
\hline Grupo Experimento & 2 dias & $10,07 \mathrm{mmHg}$ & $87,60 \%$ \\
& 7 dias & $5,07 \mathrm{mmHg}$ & $83,70 \%$ \\
& 14 dias & $3,30 \mathrm{mmHg}$ & $101,40 \%$ \\
& 21 dias & $4,00 \mathrm{mmHg}$ & $102,80 \%$ \\
28 dias & $3,50 \mathrm{mmHg}$ & $105,30 \%$ \\
& 40 dias & $2,57 \mathrm{mmHg}$ & $113,50 \%$ \\
\hline Coeficiente de correlação & & $\mathrm{r}=-0,720$ \\
Teste de significância (estatística do teste) & $\mathrm{t}=-2,075$ \\
Valor crítico (nível de significância) & & $\mathrm{tc}=-2,132$
\end{tabular}

TABELA 5 - Correlação entre manometria e edema.

\begin{tabular}{lllr}
\hline Grupos & $\begin{array}{l}\text { Tempo de } \\
\text { Aferição }\end{array}$ & Manometria & $\begin{array}{r}\text { Quantificação } \\
\text { do edema }\end{array}$ \\
\hline Grupo Experimento & 2 dias & $10,07 \mathrm{mmHg}$ & $1265,1 \mu$ \\
& 7 dias & $5,07 \mathrm{mmHg}$ & $641,1 \mu$ \\
& 14 dias & $3,30 \mathrm{mmHg}$ & $404,1 \mu$ \\
& 21 dias & $4,00 \mathrm{mmHg}$ & $157,5 \mu$ \\
& 28 dias & $3,50 \mathrm{mmHg}$ & $182,9 \mu$ \\
& 40 dias & $2,57 \mathrm{mmHg}$ & $173,0 \mu$ \\
\hline Coeficiente de correlação & $\mathrm{r}=+0,950$ \\
Teste de significância (estatística do teste) & $\mathrm{t}=+6,085$ \\
Valor crítico (nível de significância) & $\mathrm{tc}=+2,132$
\end{tabular}

maiores valores pressóricos avaliados foram acompanhados de perda de peso. Nos subgrupos de 14,21, 28 e 40 dias, mesmo com diminuição dos valores pressóricos, sem retorno aos valores do grupo controle, houve ganho de peso. Esta comparação impossibilitou estabelecimento de correlação linear significativa, uma vez que, a partir dos 14 dias, a pressão no EEI não exerceu influência na perda de peso, devido a provável adaptação do animal aos novos padrões pressóricos.

\section{Conclusões}

Após fundoplicatura de Nissen em ratos , a pressão no esfíncter esofágico inferior não retorna aos níveis do controle até o $40^{\circ}$ dia pós-operatório.

A quantificação do edema local apresenta relação diretamente proporcional à pressão no esfíncter esofágico inferior e inversamente proporcional ao peso do animal. $\mathrm{O}$ edema correlacionado à perda de peso e ao aumento da pressão no esfíncter esofágico inferior pode ser a principal variável relacionada à possível disfagia em ratos submetidos a fundoplicatura tipo Nissen.

\section{Referências}

1. Stein HJ, Barlow AP, DeMeester TR, Hinder RA. Complications of gastroesophageal reflux disease. Role of the lower esophageal sphincter, esophageal acid and acid/alkaline exposure, and duodenogastric reflux. Ann Surg 1992;216:35-43.

2. Horgan S, Pellegrini CA. Surgical treatment of gastroesophageal reflux disease. Surg Clin N Am 1997;77:1063-82.

3. Peters JH, Heimbucher J, Kauer WKH. Clinical and physiologic comparison of laparoscopic and open Nissen fundoplication. J Am Coll Surg 1995;180:385-93.

4. Spechler SJ. Epidemiology and natural history of gastroesophageal reflux disease. Digestion 1992; 51(1):24-9.

5. Nyhus LM, Condin RE. Hérnia. 3ed. Buenos Aires: Médica Panamericana; 1991. p56591.

6. Otero TZ, Jarquin AM, Ibarguen JS, Colombo LA. Complicaciones de la cirurgía antirreflujo. Cir \& Cir 2001;69(2):56-61.

7. Bais JE, Wijnhoven BPL, Masclee AAM, Smout AJPM, Gooszen HG. Analysis and surgical treatment of persistent dysphagia after Nissen fundoplication. Br J Surg 2001;88:569-76.

8. Wills VL, Hunt DR. Dysphagia after antireflux surgery. Br J Surg 2001;88:48699.

9. Pohl D, Eubanks TR, Omelanczuk PE, Pellegrini CA. Management and outcome of complications after laparoscopic antireflux operations. Arch Surg 2001;136:399-408.

10. Soto BC, Baoquan Q, Díez-Pardo J, Tovar LJA. Manometric study of the effects of experimental fundoplication in rats. Rev Esp Enferm Dig 1998;90(7):487-98.

11. Rantanen TK, Salminen JT, Makinen JE, Sipponen PI, Kiviluoto TA, Salo JÁ. Clinical significance of esophageal histologic findings after antireflux surgery. Arch Surg 2001;136:733-36. 
Rocha SL, Souza Filho ZA, Borges FF, Stroparo G, Grippa MM, Gonçalves PCZ. Experimental model of gastricesophageal fundoplication in rats: manometric and histological study of the esophagus. Acta Cir Bras [serial online] 2004 Mar-Apr;19(2). Available from URL: http://www.scielo.br/acb.

ABSTRACT - Purpose: The aim of this experimental study is to evaluate the outcomes of having Nissen fundoplication done on rats through the histological analysis of the distal esophagus and manometric study of the lower esophageal sphincter (LES), as well as the evaluation of weight variation of the rats subjected to this procedure, in distinct days after surgery. Methods: Have been used 70 male Wistar rats, at the ages of 100 to 130 days, weighing from 250 to 300 grams and divided into 2 groups. The experiment group was composed of 60 rats, subdivided into 6 subgroups according to the time of post-operatory manometric measurement: 02, 07, 14, 21, 28 and 40 days, and subjected to fundoplication with posterior manometric analysis of the LES and histological analysis of the distal esophagus. The control group was composed of the ten left animals, which were subjected only to laparotomy with manipulation of the gastric-esophageal junction, and the same analysis has been performed. Results: Histological analysis evidenced statistically significant edema $(\mathrm{p}=0,0000)$ accompanied of weight loss in the subgroups 02 and 07 days. Significant statistic in the manometric study of all groups compared to the control group has been observed $(\mathrm{p}=0,0018)$. The edema and weight correlation was linear significant and inversely proportional $\left(t=2,577<t_{c}=+2,132\right)$. Sgnificant linear and directly proportional correlation between edema and manometric has been established $\left(t=+6,085>t_{c}=\right.$ $+2,132$ ). Conclusion: After Nissen procedure in rats, the pressure in the LES does not return to the levels of control group until the $40^{\circ}$ post-operatory day. The edema correlated with weight loss and pressure rise in the LES can be the main variable related to the possible dysphagia.

KEY WORDS - Fundoplication. Dysphagia. Edema. Manometry.

Correspondência:

Sérgio Luiz Rocha

Av. Iguaçu, 3000/2001

80240-031 Curitiba-PR

rocha@bbs2.sul.com.br

Conflito de interesse: nenhum

Fonte de financiamento: nenhuma

Data da revisão: $15 / 01 / 2004$

Data da aprovação: 01/02/2004

*Fotos coloridas disponíveis em www.scielo.br/acb 